\title{
BMJ
}

\section{Spontaneous preterm birth and small for gestational age infants in women who stop smoking early in pregnancy: prospective cohort study}

\author{
Lesley M E McCowan, associate professor of obstetrics and gynaecology, ${ }^{1}$ Gustaaf A Dekker, professor of \\ obstetrics and gynaecology, ${ }^{6}$ Eliza Chan, research fellow, ${ }^{1}$ Alistair Stewart, statistician, ${ }^{2}$ Lucy C Chappell, \\ senior lecturer in maternal and fetal medicine, ${ }^{4}$ Misty Hunter, medical student, ${ }^{1}$ Rona Moss-Morris, professor \\ of health psychology, ${ }^{5}$ Robyn A North, professor in obstetric medicine ${ }^{3}$ On behalf of the SCOPE consortium
}

Department of Obstetrics and Gynaecology, Faculty of Medical and Health Sciences, School of Population Health, University of Auckland, Auckland, New Zealand

${ }^{2}$ Department of Epidemiology and Biostatistics, Faculty of Medical and Health Sciences, School of Population Health, University of Auckland, Auckland, New Zealand

${ }^{3}$ Discipline of Obstetrics and Gynaecology, School of

Paediatrics and Reproductive Health, University of Adelaide, Australia

${ }^{4}$ Division of Reproduction and Endocrinology, King's College London

${ }^{5}$ School of Psychology, University of Southampton

${ }^{6}$ Women and Children's Division, Lyell McEwin Hospital, University of Adelaide, Adelaide, South Australia

Correspondence to:

L M E McCowan,

l.mccowan@auckland.ac.nz

Cite this as: BMJ 2009;338:b1081 doi:10.1136/bmi.b1081

\section{ABSTRACT}

Objectives To compare pregnancy outcomes between women who stopped smoking in early pregnancy and those who either did not smoke in pregnancy or continued to smoke.

Design Prospective cohort study.

Setting Auckland, New Zealand and Adelaide, Australia.

Participants 2504 nulliparous women participating in the Screening for Pregnancy Endpoints (SCOPE) study grouped by maternal smoking status at $15( \pm 1)$ week's gestation.

Main outcome measures Spontaneous preterm birth and small for gestational age infants (birth weight $<10$ th customised centile). We compared odds of these outcomes between stopped smokers and non-smokers, and between current smokers and stopped smokers, using logistic regression, adjusting for demographic and clinical risk factors.

Results $80 \%$ ( $n=1992)$ of women were non-smokers, $10 \%$ $(n=261)$ had stopped smoking, and $10 \%(n=251)$ were current smokers. We noted no differences in rates of spontaneous preterm birth $(4 \%, n=88 v 4 \%, n=10$; adjusted odds ratio $1.03,95 \%$ confidence interval 10.49 to $2.18 ; \mathrm{P}=0.66)$ or small for gestational age infants $(10 \%$, $\mathrm{n}=195 \vee 10 \%, \mathrm{n}=27 ; 1.06,0.67$ to $1.68 ; \mathrm{P}=0.8$ ) between non-smokers and stopped smokers. Current smokers had higher rates of spontaneous preterm birth $(10 \%, n=25 \mathrm{~V}$ $4 \%, n=10 ; 3.21,1.42$ to $7.23 ; P=0.006)$ and small for gestational age infants $(17 \%, n=42 \vee 10 \%, n=27 ; 1.76$, 1.03 to 3.02; $\mathrm{P}=0.03$ ) than stopped smokers.

Conclusion In women who stopped smoking before 15 weeks' gestation, rates of spontaneous preterm birth and small for gestational age infants did not differ from those in non-smokers, indicating that these severe adverse effects of smoking may be reversible if smoking is stopped early in pregnancy.

\section{INTRODUCTION}

A causal and dose dependent relationship has been established between smoking during pregnancy and the risk of having a small for gestational age infant and of spontaneous preterm birth. ${ }^{12}$ Women who smoke also have raised risks of pregnancy loss with increased rates of miscarriage, ectopic pregnancy, stillbirth, and neonatal death. ${ }^{2-4}$ The costs of maternal and infant complications due to smoking during pregnancy are enormous, with costs for the United States estimated to exceed $\$ 1.4 \mathrm{bn}$ ( $£ 1 \mathrm{bn}, € 1.1 \mathrm{bn})$ in $1995 .^{5}$ More than $90 \%$ of the costs for infants are attributable to care for those with low birth weight in the neonatal period and the first year of life. ${ }^{6}$ From a population perspective, smoking is the most modifiable risk factor for adverse pregnancy outcomes in developed countries.

Although stopping smoking in pregnancy has been known for some time to reduce the rate of low birthweight infants and total preterm births, ${ }^{7}$ very few studies have addressed whether there is a critical gestation time by which smoking must stop to prevent smoking induced complications of pregnancy. ${ }^{89}$ Surprisingly, no studies to date have specifically addressed whether stopping smoking in early pregnancy reduces the rate of spontaneous preterm birth or small for gestational age infants. If a critical period of gestation was identified by which smoking needed to cease to reduce these severe complications, such data would have important public health implications for pregnant women and their maternity care providers.

Our objective was to compare rates of spontaneous preterm birth and small for gestational age infants in women who stopped smoking before $15( \pm 1)$ weeks' gestation with those in women who did not smoke in pregnancy, and with those in women who continued to smoke. A secondary objective was to compare measures of stress, anxiety, and depression between the three smoking groups. We hypothesised that rates of spontaneous preterm birth and small for gestational age infants would not differ between women who stopped smoking by $15( \pm 1)$ weeks' gestation and non-smokers, and that women who continued to smoke would have increased rates of spontaneous preterm birth and small for gestational age infants 
compared with those who stopped smoking by $15( \pm 1)$ weeks' gestation.

\section{METHODS}

The participants were healthy nulliparous women with singleton pregnancies recruited to the Screening for Pregnancy Endpoints (SCOPE) study between November 2004 and July 2007 in Auckland, New Zealand, and Adelaide, Australia. ${ }^{10}$ SCOPE is a prospective, multicentre cohort study with the main aim of developing screening tests to predict pre-eclampsia, small for gestational age infants, and spontaneous preterm birth.

Women were recruited to the SCOPE study by 15 weeks' gestation through hospital antenatal clinics, obstetricians, general practitioners, community midwives, and self referral in response to advertisements or recommendations of friends. Women were excluded if they were judged to be at high risk of preeclampsia, small for gestational age babies, or spontaneous preterm birth because of underlying medical conditions, gynaecological history, three or more previous miscarriages, or three or more terminations of pregnancy, or who had received interventions that might modify pregnancy outcome. ${ }^{10}$

A research midwife interviewed and examined women who agreed to participate at 15 and 20 weeks' gestation. At the interview women also completed a lifestyle questionnaire incorporating the following psychological scales: the short form of the state trait anxiety index, measuring anxiety; ${ }^{11}$ the perceived stress scale, measuring perceived stress; ${ }^{12}$ and the Edinburgh postnatal depression scale, measuring depression. ${ }^{13}$ We use data from the 15 weeks' interview in this publication. At the time of interview, data were entered into an internet accessed, central database that has a complete audit trail. Participants were followed up prospectively, with pregnancy outcome data and baby measurements collected by research midwives, usually within 72 hours of birth. Stringent data monitoring included individual checking of all data for each participant (including checking for any data entry errors in the lifestyle questionnaire) and using a customised software program to detect any systematic data entry errors.

Participants were divided into three groups, according to self reported maternal smoking status, which was ascertained by direct questioning at recruitment to the SCOPE study at 15 weeks' gestation. "Non-smoker" referred to women who did not smoke at any time during pregnancy, "stopped smoker" to women who had smoked at some time during pregnancy but had stopped before the 15 weeks' SCOPE interview, and "current smoker" to women who continued to smoke at the 15 weeks' interview. Stopped smokers were asked at what gestation they stopped smoking, and stopped and current smokers were asked about the number of cigarettes smoked daily in the three months before pregnancy, in the first trimester, and at the 15 weeks' SCOPE visit, as appropriate. Smoking status was not confirmed using biochemical measures.

\section{Outcome measures}

The primary outcome measures were spontaneous preterm birth and small for gestational age by customised centiles. Secondary outcome measures were birth weight, gestation at delivery, uncomplicated pregnancy, and measures of stress, anxiety, and depression.

\section{Definitions}

The estimated date of delivery was calculated from a certain last menstrual period date. The estimated date of delivery was only adjusted if either a scan at less than 16 weeks' gestation found a difference of seven or more days between the scan gestation and that calculated by the last menstrual period, or at a 20 week scan a difference of 10 or more days was found between the scan gestation and that calculated from the last menstrual period. If the last menstrual period date was uncertain, scan dates were used to calculate the estimated date of delivery. Small for gestational age was defined as birth weight below the 10th customised centile adjusted for maternal weight, height, parity, ethnic group, and infant sex (www.gestation.net). ${ }^{14}$ Spontaneous preterm birth was defined as spontaneous preterm labour or preterm premature rupture of the membranes resulting in preterm birth at less than 37 weeks' gestation. Pre-eclampsia was defined as gestational hypertension (systolic blood pressure $\geq 140 \mathrm{mmHg}$ and/or diastolic blood pressure $\geq 90 \mathrm{mmHg}$ on at least two occasions four hours apart after 20 weeks' gestation, but before the onset of labour, or postpartum systolic blood pressure $\geq 140 \mathrm{mmHg}$ and/or diastolic blood pressure $\geq 90 \mathrm{mmHg}$ on at least two occasions four hours apart) with proteinuria (24 hour urinary protein $\geq 300 \mathrm{mg}$, or spot urine protein to creatinine ratio $\geq 30 \mathrm{mg} / \mathrm{mmol}$ creatinine, or urine dipstick protein $\geq 2$ + ) or any multisystem complication of pre-eclampsia. ${ }^{15}$ Uncomplicated pregnancy was defined as a pregnancy with no antenatal obstetric or medical complications and resulting in delivery of an appropriately grown, healthy baby at 37 or greater weeks' gestation.

\section{Statistical methods}

We used ANOVA tests to compare continuous variables between the three smoking groups with post hoc Tukey test for pairwise comparisons, Student's $t$ test was used to compare continuous variables between stopped smokers and non-smokers and between stopped smokers and current smokers, and $\chi^{2}$ or Fisher's exact tests to compare categorical variables, as appropriate.

We used logistic regression to compare the odds of spontaneous preterm birth and small for gestational age between stopped smokers and non-smokers and between stopped smokers and current smokers, adjusting for potential confounders. Potential confounders included demographic factors (age, self assigned ethnic group, marital status, employment, and body mass index), risk factors suggested in previous publications (bleeding during pregnancy, folic acid use, use of 
multivitamins or alcohol at 15 weeks gestation, and depression, stress, and anxiety scores at 15 weeks' gestation). The logistic regression model for spontaneous preterm birth also included history of previous miscarriage or termination of pregnancy and large loop excision of the cervical transformation zone. All variables were obligatory data points and were available for all of the participants, with the exception of scores for the psychological scales, which were missing in $18(0.7 \%)$ of the participants. We excluded data for these 18 women from the logistic regression models.

\section{RESULTS}

Between November 2004 and July 2007, 2535 women were recruited to the SCOPE study in Auckland and

\begin{tabular}{|c|c|c|c|c|}
\hline & $\begin{array}{c}\text { Non-smokers } \\
(\mathrm{n}=1992,80 \%)\end{array}$ & $\begin{array}{c}\text { Stopped } \\
\text { smokers } \\
(n=261,10 \%)\end{array}$ & $\begin{array}{c}\text { Current } \\
\text { smokers } \\
(n=251,10 \%)\end{array}$ & $P$ value \\
\hline Age (years) & $29.7(5.1)$ & $25.2(5.9)$ & $23.1(5.5)$ & $<0.001^{*} † \ddagger$ \\
\hline Ethnic origin & $1725(87)$ & $223(85)$ & $225(90)$ & $<0.001$ \\
\hline European & $107(5)$ & $9(3)$ & $3(1)$ & \\
\hline Asian & $67(3)$ & $1(\ll 1)$ & $0(0)$ & \\
\hline Indian & $57(3)$ & $22(8)$ & $15(6)$ & \\
\hline Polynesian & $36(2)$ & $6(2)$ & $8(3)$ & \\
\hline Married/defacto & $1912(96)$ & $213(82)$ & $188(75)$ & $<0.001$ \\
\hline Single & $80(4)$ & $48(18)$ & $63(25)$ & \\
\hline Schooling $\leq 12$ years & $771(39)$ & $163(63)$ & $198(79)$ & $<0.001$ \\
\hline Full/part time work & $1791(90)$ & $198(76)$ & $144(57)$ & $<0.001$ \\
\hline No paid work & $201(10)$ & $63(24)$ & $107(43)$ & \\
\hline \multicolumn{5}{|l|}{ Body mass index $\left(\mathrm{kg} / \mathrm{m}^{2}\right)$} \\
\hline$<20.0$ & $139(7)$ & $17(7)$ & $41(16)$ & $<0.001$ \\
\hline $20.0-24.9$ & $1012(51)$ & $115(44)$ & $95(38)$ & \\
\hline $25.0-29.9$ & $561(28)$ & $73(28)$ & $57(23)$ & \\
\hline$\geq 30.0$ & $280(14)$ & $56(21)$ & $58(23)$ & \\
\hline Any previous pregnancy & $473(24)$ & $78(30)$ & $85(34)$ & $<0.001$ \\
\hline \multicolumn{5}{|l|}{ Miscarriage } \\
\hline 0 & $1735(87)$ & $227(87)$ & $208(83)$ & 0.35 \\
\hline$\geq 1$ & 257 (13) & 34 (13) & $43(17)$ & \\
\hline \multicolumn{5}{|l|}{ Termination of pregnancy } \\
\hline 0 & $1750(88)$ & $211(81)$ & $198(79)$ & $<0.001$ \\
\hline 1 & $215(11)$ & $38(15)$ & $42(17)$ & \\
\hline$\geq 2$ & $27(1)$ & $12(5)$ & $11(4)$ & \\
\hline LLETZ treatment & $85(4)$ & $8(3)$ & $12(5)$ & 0.59 \\
\hline \multicolumn{5}{|l|}{ Current pregnancy } \\
\hline Vaginal bleeding & $423(21)$ & $52(20)$ & $44(18)$ & 0.37 \\
\hline Alcoholई & $87(4)$ & $16(6)$ & $20(8)$ & 0.03 \\
\hline Folic acid§ & $1492(75)$ & $189(72)$ & $163(65)$ & 0.003 \\
\hline Multivitamins§ & $1178(59)$ & $132(51)$ & $102(41)$ & $<0.001$ \\
\hline \multicolumn{5}{|l|}{ Psychological scales } \\
\hline Depression & $6.4(4.3)$ & $8.7(5.2)$ & $9.0(6.2)$ & $<0.001^{*} \dagger$ \\
\hline Anxiety & $32.4(10.6)$ & $35.2(12.2)$ & $36.9(13.5)$ & $<0.001^{*} \dagger$ \\
\hline Perceived stress & $14.0(5.9)$ & $16.8(6.8)$ & $17.5(7.4)$ & $<0.001^{*} \dagger$ \\
\hline \multicolumn{5}{|c|}{$\begin{array}{l}\text { Data are mean (SD) or number \%. P values are for comparisons between the three smoking groups using } x^{2} \text { or } \\
\text { analysis of variance test, with post hoc Tukey test for pairwise comparisons, P<0.05. LLETZ=large loop excision } \\
\text { of transformation zone. } \\
\text { *Non-smokers } v \text { current smokers. } \\
\text { †Non-smokers } v \text { stopped smokers. } \\
\text { †Stopped smokers } v \text { current smokers. } \\
\text { §Using at } 15 \text { week interview. }\end{array}$} \\
\hline
\end{tabular}

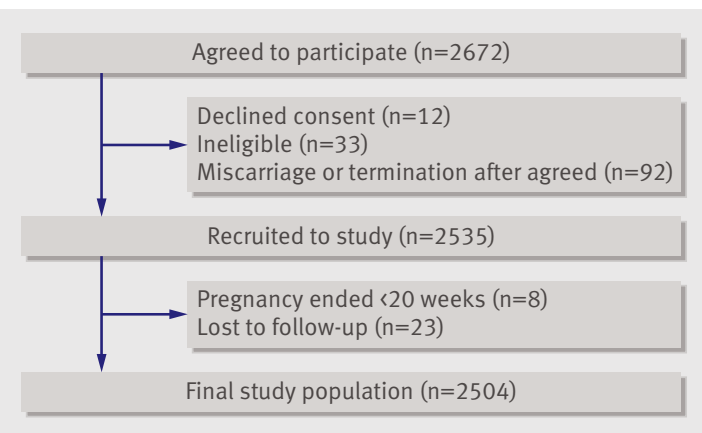

Fig 1| Participants recruited

Adelaide and follow up was complete in 99\% of participants ( $\mathrm{n}=2504$, fig 1$)$. At the interview at 15 weeks' gestation, $80 \%(n=1992)$ of women reported that they were non-smokers, $10 \%(\mathrm{n}=261)$ had stopped smoking during pregnancy, and $10 \%(\mathrm{n}=251)$ had continued to smoke. Of the stopped smokers, 51\% (n=134) stopped smoking before 6 weeks' gestation, $43 \% \quad(n=112)$ stopped between 6 and 12 weeks, and $6 \%(n=15)$ stopped after 12 weeks' gestation but before 15 weeks. The mean number of cigarettes smoked per day before pregnancy was greater in women who continued to smoke $(17.8,95 \%$ confidence interval 16.7 to 18.9$)$ than in those who stopped $(8.9,8.0$ to 9.8; $\mathrm{P}<0.001)$. Of continued smokers, $49 \%(\mathrm{n}=122)$ reported they currently smoked between one and five cigarettes daily, $31 \%(n=79)$ smoked six to 10 cigarettes, and $20 \%(\mathrm{n}=50)$ smoked more than 10 cigarettes daily.

Background characteristics differed between the three smoking groups (table 1). Current smokers were younger, were more likely to be single, had fewer years of schooling, and were less likely to be employed than non-smokers. Women with low $(<20)$ and high $(>30)$ body mass index values seemed to be more common in the smoking group compared to nonsmokers. Current smokers were more likely to be drinking alcohol and less likely to be taking folic acid or pregnancy multivitamins at 15 weeks' gestation. Mean scores for measures of depression, anxiety, and stress differed across the three smoking groups, but scores did not differ between current and stopped smokers (table 1).

We noted no differences between stopped smokers and non-smokers, respectively, in rates of spontaneous preterm birth $(4 \%, \mathrm{n}=10 v 4 \%, \mathrm{n}=88 ; \mathrm{P}=0.66)$ or small for gestational age infants $(10 \%, \mathrm{n}=27 \vee 10 \%, \mathrm{n}=195$; $\mathrm{P}=0.80$; table 2). Compared with stopped smokers, current smokers had significantly higher rates of spontaneous preterm birth $(10 \%, \mathrm{n}=25$ v $4 \%, \mathrm{n}=10$; $\mathrm{P}=0.006)$ and small for gestational age infants $(17 \%$, $\mathrm{n}=42$ v $10 \%, \mathrm{n}=27 ; \mathrm{P}=0.03)$. Women who stopped smoking had higher rates of uncomplicated pregnancies than women who continued to smoke $(62 \%, \mathrm{n}=162$ v 44\%, $\mathrm{n}=111 ; \mathrm{P}<0.001)$.

Logistic regression analysis adjusting for potential confounders (demographic and clinical risk factors) confirmed that the rates of spontaneous preterm birth 
(adjusted odds ratio 1.03, 95\% confidence interval 0.49 to $2.18 ; \mathrm{P}=0.93$ ) and small for gestational age infants (1.06, 0.67 to $1.68 ; \mathrm{P}=0.81$ ) were very similar between stopped smokers and non-smokers. The adjusted odds ratio for spontaneous preterm birth (3.21, 1.42 to 7.23; $\mathrm{P}=0.005)$ or small for gestational age infants $(1.76,1.03$ to $3.02 ; \mathrm{P}=0.04)$ remained significantly raised in current smokers compared with women who stopped smoking.

\section{DISCUSSION}

In this large prospective cohort study of healthy nulliparous women, those who reported that they had stopped smoking before 15 weeks' gestation had rates of spontaneous preterm birth, small for gestational age infants, and uncomplicated pregnancies similar to those in non-smokers. A cause for concern was that after adjusting for other risk factors, women who continued to smoke at 15 weeks' gestation had three times the rate of spontaneous preterm birth and almost double the rate of small for gestational age infants compared with women who stopped smoking. Our results are of considerable public health importance. The data suggest that the adverse effects of smoking on these late pregnancy outcomes may be largely reversible if smoking is stopped early in pregnancy, offering an important incentive for pregnant women who smoke to stop smoking early in pregnancy.

\section{Strengths and weaknesses}

The strengths of our study are that detailed information about smoking status was collected prospectively, pregnancy outcome data were available in more than $99 \%$ of participants, and pregnancy outcome was assigned according to pre-specified criteria. Stringent data monitoring protocols ensured the quality of the data.

A potential limitation is that, as is usual in clinical practice, self reported smoking status was not validated by measurement of cotinine levels. Cotinine measurements are highly correlated with self reported smoking status, only account for slightly more variation in birth weight than smoking history, ${ }^{16}$ and have other limitations. ${ }^{16-18}$ In view of the marked similarities in pregnancy outcomes between stopped smokers and non-smokers, it is unlikely that many women who continued to smoke claimed to have stopped smoking.

\section{Comparison with other studies}

Published data about rates of preterm birth in relation to the gestation at which smoking ceased are very limited. Preterm birth may result from either spontaneous onset of preterm labour or iatrogenic preterm birth for maternal or fetal indications. $\mathrm{Li}$ and colleagues reported that women who stopped smoking by 32 weeks' gestation had increased mean gestation at delivery and reduced total preterm births compared with continued smokers, but provided no data about spontaneous preterm birth. ${ }^{19}$ Our results that women who stop smoking in early pregnancy have rates of spontaneous preterm birth similar to non-smokers are compatible with data showing that women with a previous preterm birth, who stopped smoking in between pregnancies, reduced their risk of spontaneous preterm birth in the next pregnancy to that of non-smokers. ${ }^{20}$

Surprisingly, no studies have reported the relation between gestational age at cessation of smoking during pregnancy and rates of small for gestational age infants. Two early publications assessed birth weight and perinatal mortality in relation to whether smoking was stopped early in pregnancy. ${ }^{89}$ Butler and colleagues retrospectively recorded smoking history after delivery from participants in the British Perinatal Mortality Surveys in 1963 and 1969 (n=16994 live births and 6890 perinatal deaths). ${ }^{8}$ Our data are consistent with their finding that perinatal mortality and mean infant birth weight in women who never smoked in pregnancy were virtually identical to those in women who reported stopping smoking by four months' gestation. Another large study based in the United Kingdom 20 years ago investigated the relation between birth weight and maternal smoking status at six weeks, at 16 weeks, and after 16 weeks' gestation. ${ }^{9}$ Women who reported stopping smoking by six weeks and also between six and 16 weeks' gestation had infants with mean birth weight similar to that of non-smokers

Table 2|Pregnancy outcomes

\begin{tabular}{|c|c|c|c|c|c|c|c|}
\hline & $\begin{array}{c}\text { Non- } \\
\text { smokers } \\
(n=1992)\end{array}$ & $\begin{array}{l}\text { Stopped } \\
\text { smokers } \\
(n=261)\end{array}$ & $\begin{array}{l}\text { Mean difference } \\
(95 \% \mathrm{Cl}) *\end{array}$ & P value* & $\begin{array}{l}\text { Current } \\
\text { smokers } \\
(n=251)\end{array}$ & $\begin{array}{l}\text { Mean difference } \\
(95 \% \mathrm{Cl}) \dagger\end{array}$ & $\mathrm{P}$ value $\dagger$ \\
\hline $\begin{array}{l}\text { Spontaneous preterm } \\
\text { birth }\end{array}$ & $88(4)$ & $10(4)$ & $-0.6 \%(-2.6$ to 2.6$)$ & 0.66 & $25(10)$ & $6.1 \%(1.7$ to 10.8$)$ & 0.006 \\
\hline Small forgestational age & $195(10)$ & $27(10)$ & $-0.5 \%(-5.0$ to 2.9$)$ & 0.80 & $42(17)$ & $6.4 \%$ (0.4 to 12.4 & 0.03 \\
\hline Birth weight (g) & $3409(592)$ & $3479(560)$ & $-70(-146$ to 6$)$ & 0.09 & $3139(751)$ & 270 (190 to 350$)$ & $<0.001$ \\
\hline $\begin{array}{l}\text { Delivery gestation } \\
\text { (weeks) }\end{array}$ & $39.5(2.3)$ & $39.7(2.4)$ & $-0.2(-0.5$ to 0.1$)$ & 0.11 & $38.6(3.6)$ & 0.9 (0.6 to 1.2$)$ & $<0.001$ \\
\hline Customised centile & $48.9(28.7)$ & $49.3(28.5)$ & $-0.4(-4.1$ to 3.3$)$ & 0.88 & $41.3(29.7)$ & 7.6 (3.8 to 11.4$)$ & 0.002 \\
\hline $\begin{array}{l}\text { Uncomplicated } \\
\text { pregnancy }\end{array}$ & $1192(60)$ & $162(62)$ & $-2.2 \%(-8.3$ to 4.2$)$ & 0.49 & $111(44)$ & $-17.8 \%(-26.1$ to -9.2$)$ & $<0.001$ \\
\hline
\end{tabular}

Data are mean (SD) or number (\%). $\mathrm{P}$ values are for $\mathrm{X}^{2}$ or Student's $t$ test.

*Comparison between stopped smokers and non-smokers.

†Comparison between stopped smokers and current smokers. 
whereas women who stopped smoking after 16 weeks had infants with reduced mean birth weight.

These previous studies investigated the effect of timing of smoking cessation on birth weight, which is affected by gestational age at delivery as well as fetal growth. A novel feature of our study is that we have provided specific data about small for gestational age infants. We defined small for gestational age using customised birthweight centiles, which better identify small infants at higher risk of morbidity and mortality than raw birth weight or definition of small for gestational age by a population reference. ${ }^{2122}$

When considered with previous reports, our data indicate that stopping smoking early in pregnancy, and certainly by 15-16 weeks' gestation, may minimise the adverse effects of smoking on late pregnancy complications and should be an important goal for pregnant smokers. Our findings provide further support for the recommendation from the National Institute of Health and Clinical Excellence (NICE) antenatal care guideline that women should have an appointment with a maternity care provider by 10 weeks' gestation to receive "lifestyle advice including smoking cessation." 20

Many studies have found an association between depression, psychosocial stress, and current smoking. ${ }^{234}$ Both depression and high levels of perceived stress have been associated with a decreased likelihood of quitting smoking in women who are not pregnant. ${ }^{2425}$ The two studies addressing the relation between scores for anxiety, perceived stress or depression, and cessation of smoking in pregnant women have conflicting results. In the first, socially disadvantaged women who stopped smoking during pregnancy were more likely than continued smokers to score in the distressed range of the Mental Health Questionnaire. ${ }^{26}$ By contrast, a more recent study reported that women who stopped smoking in early pregnancy had lower levels of stress and depressive symptoms than continued smokers. ${ }^{27}$ Women who ceased smoking in our study did not have increased measures of stress, anxiety, or depression compared with current smokers, suggesting that smoking cessation did not increase psychological morbidity. This finding should reassure pregnant women who may have the misconception that stopping smoking in pregnancy will increase their stress levels.

Several smoking cessation interventions, including cognitive behavioural therapy and rewards with social support, reduce smoking during pregnancy and improve pregnancy outcome. ${ }^{7}$ However, a substantial proportion of women continue to smoke despite these programmes and most interventions focus specifically on altering smoking behaviour. The finding that both stopped and current smokers have higher levels of distress on all dimensions measured (anxiety, depression, and perceived stress) than non-smokers suggests that strategies to help women manage this distress merit consideration in future pregnancy smoking cessation strategies.
Social inequalities have increased over time between women who smoke and non-smokers, ${ }^{28}$ and the demographic characteristics of pregnant women, categorised by smoking status, in our study are consistent with those in other reports. ${ }^{29}$ Women who continued to smoke were heavier smokers before pregnancy, younger, less well educated, and less likely to be employed, and reported higher rates of alcohol use. Those who stopped by 15 weeks' gestation had rates of these characteristics intermediate between non-smokers and smokers. We therefore consider the results of this study are likely to be generalisable to other groups of pregnant women.

Our data should not be misinterpreted as a justification to reduce efforts to assist pregnant women to strive to become smoke free if they still smoke after 16 weeks' gestation. Improved pregnancy outcomes have been reported in women who stop by as late as 32 weeks' gestation. ${ }^{19}$ Moreover, stopping smoking at any gestation in pregnancy, if sustained in the postpartum period, has enormous additional benefits on newborn and child health. ${ }^{2}$

\section{Conclusion and policy implications}

Data from this large prospective cohort study of nulliparous women have shown that stopping smoking before 15 weeks' gestation is associated with rates of spontaneous preterm birth and small for gestational age infants similar to those in women who do not smoke in pregnancy. Maternity care providers should strive to assist pregnant women who smoke to stop early in pregnancy, emphasising the major health benefits if they cease to smoke before 15 weeks' gestation.

Acknowledgments: We thank the pregnant women who participated in the SCOPE study, Claire Roberts for her contributions in establishing the SCOPE study in Adelaide, Rennae Taylor for coordinating the New Zealand SCOPE study and assistance with manuscript preparation, Denise Healy for coordinating the Australian SCOPE study, the SCOPE

\section{WHAT IS ALREADY KNOWN ON THIS TOPIC}

- Smoking is the single most modifiable risk factor for adverse pregnancy outcomes in developed countries

- Stopping smoking in pregnancy increases birth weight and reduces rates of all preterm birth

- The gestation by which smoking must stop to reverse effects of smoking on spontaneous preterm births and small for gestational age infants is not known

\section{WHAT THIS PAPER ADDS}

- Stopping smoking early in pregnancy, before 15 weeks' gestation, results in rates of spontaneous preterm births and small for gestational age infants similar to those in non-smokers

- Women who continue to smoke at 15 weeks' gestation are more likely than those who stop smoking to have spontaneous preterm birth

- Pregnant women should be offered support and interventions to help them stop smoking early in pregnancy 
research midwives, and Hayden McRobbie for helpful comments on the manuscript.

Contributors: LMEMCC is guarantor. LMEMCC, GAD, LCC, MH, RAN, and RMM had a role in conception and design. LMEMCC, GAD, EC, AS, LCC, RAN, and RMM interpreted data. All authors took part in drafting the article or revising it for critically important intellectual content and all gave final approval of the version to be published.

Funding: New Zealand SCOPE Study-New Enterprise Research Fund Foundation for Research Science and Technology; Health Research Council; Evelyn Bond Fund, Auckland District Health Board Charitable Trust. Australian SCOPE Study-Premier's Science and Research Fund, South Australian Government. The study sponsors had no role in study design, data analysis, or writing this report.

Competing interests: None declared.

Ethical approval: Ethical approval was gained from local ethics committees (New Zealand AKX/02/00/364 and Australia REC 1712/5/ 2008) and all women provided written informed consent.

1 Kramer MS. Determinants of low birth weight: methodological assessment and meta-analysis. Bull World Health Organ 1987;65:663-737.

2 Cnattingius S. The epidemiology of smoking during pregnancy: smoking prevalence, maternal characteristics, and pregnancy outcomes. Nicotine Tob Res 2004;6(suppl 2):S125-40.

3 Andres RL. Perinatal complications associated with maternal smoking. Sem Neonatol 2005;5:231-41.

4 Castles A, Adams EK, Melvin CL, Kelsch C, Boulton ML. Effects of smoking during pregnancy. Five meta-analyses. Am J Prev Med 1999;16:208-15.

5 Centers for Disease Control. Medical-care expenditure attributable to cigarette smoking-United States,1995. MMWR Morb Mortal Wkly Rep 1997;46:1048-50.

6 Miller DP, Villa KF, Hogue SL, Sivapathasundaram D. Birth and firstyear costs for mothers and infants attributable to maternal smoking. Nicotine Tob Res 2001;3:25-35.

7 Lumley J, Oliver SS, Chamberlain C, Oakley L. Interventions for promoting smoking cessation during pregnancy. Cochrane Database of Systematic Reviews 2004:CD001055.

8 Butler NR, Goldstein H, Ross EM. Cigarette smoking in pregnancy: its influence on birth weight and perinatal mortality. BMJ 1972;2:127-30.

9 MacArthur C, Knox EG. Smoking in pregnancy: effects of stopping at different stages. Br J Obstet Gynaecol 1988;95:551-5.

10 McCowan L, North R, Taylor R. ACTRN12607000551493. Australian New Zealand Clinical Trials Registry, 2007. www.anzctr.org.au/ trialSearch.aspx.

11 Marteau TM, Bekker $\mathrm{H}$. The development of a six-item short-form of the state scale of the Spielberger State-Trait Anxiety Inventory (STAI) BrJ Clin Psychol 1992;31:301-6.

12 Cohen S, Kamarck T, Mermelstein R. A global measure of perceived stress. J Health Soc Behav 1983;24:385-96.
13 Peindl KS, Wisner KL, Hanusa BH. Identifying depression in the first postpartum year: guidelines for office-based screening and referral. Affect Disord 2004;80:37-44.

14 Gardosi J, Francis A. Customised centile calculator-GROW 6.12 bulk. Gestation Network, 2007.www.gestation.net/birthweight_centiles/ birthweight centiles.htm.

15 Brown MA, Hague WM, Higgins J, Lowe S, McCowan L, Oats J, et al. The detection, investigation and management of hypertension in pregnancy: executive summary. Aust N Z J Obstet Gynaecol 2000;40:133-8.

16 Secker-Walker RH, Vacek PM. Relationships between cigarette smoking during pregnancy, gestational age, maternal weight gain, and infant birthweight. Addict Behav 2003;28:55-66.

17 Benowitz NL, Hall SM, Herning RI, Jacob P 3rd, Jones RT, Osman AL. Smokers of low-yield cigarettes do not consume less nicotine. N Engl Med 1983;309:139-42.

18 Biber A, Scherer G, Hoepfner I, Adlkofer F, Heller WD, Haddow JE, et al. Determination of nicotine and cotinine in human serum and urine: an interlaboratory study. Toxicol Lett 1987;35:45-52.

19 Li CQ Windsor RA, Perkins L, Goldenberg RL, Lowe JB. The impact on infant birth weight and gestational age of cotinine-validated smokin reduction during pregnancy. JAMA 1993;269:1519-24.

20 Cnattingius S, Granath F, Petersson G, Harlow BL. The influence of gestational age and smoking habits on the risk of subsequent preterm deliveries. N Engl J Med 1999;341:943-8.

21 Ego A, Subtil D, Grange G, Thiebaugeorges O, Senat MV, Vayssiere C, et al. Customized versus population-based birth weight standards for identifying growth restricted infants: a French multicenter study. Am J Obstet Gynecol 2006;194:1042-9.

22 Zhang X, Platt R, Cnattingius S, Kramer M. The use of customised versus population-based birthweight standards in predicting perinatal mortality. BrJ Obstet Gynaecol 2007;114:474-7.

23 Fergusson DM, Goodwin RD, Horwood LJ. Major depression and cigarette smoking: results of a 21-year longitudinal study. Psychol Med 2003;33:1357-67.

24 Cohen S, Lichtenstein E. Perceived stress, quitting smoking, and smoking relapse. Health Psychol 1990;9:466-78.

25 Kendler KS, Neale MC, MacLean CJ, Heath AC, Eaves LI, Kessler RC. Smoking and major depression. A causal analysis. Arch Gen Psychiatry 1993;50:36-43.

26 Blalock JA, Robinson JD, Wetter DW, Cinciripini PM. Relationship of DSM-IV-based depressive disorders to smoking cessation and smoking reduction in pregnant smokers. Am J Addict 2006;15:268-77.

27 Ludman EJ, McBride CM, Nelson IC, Curry SJ, Grothaus LC, Lando HA et al. Stress, depressive symptoms, and smoking cessation among pregnant women. Health Psychol 2000;19:21-7.

28 Gilman SE, Breslau J, Subramanian SV, Hitsman B, Koenen KC. Social factors, psychopathology, and maternal smoking during pregnancy. Am J Public Health 2008;98:448-53.

Accepted: 1 March 2009 